Draft Version April 22, 2022

Preprint typeset using $\mathrm{L}_{\mathrm{T}}^{\mathrm{T}} \mathrm{E}$ X style emulateapj v. 5/2/11

\title{
SN 2010LP—A TYPE IA SUPERNOVA FROM A VIOLENT MERGER OF TWO CARBON-OXYGEN WHITE DWARFS
}

\author{
M. Kromer ${ }^{1,2}$, R. Pakmor ${ }^{3}$, S. Taubenberger ${ }^{1}$, G. Pignata ${ }^{4}$, M. Fink $^{5}$, F. K. Röpke ${ }^{5}$, I. R. Seitenzahl ${ }^{5,1}$, S. A. Sim $^{6}$ \\ \& W. HillebrandT ${ }^{1}$ \\ (Dated: Received ; accepted) \\ Draft version April 22, 2022
}

SN 2010lp is a subluminous Type Ia supernova (SN Ia) with slowly-evolving lightcurves. Moreover, it is the only subluminous SN Ia observed so far that shows narrow emission lines of [O I] in latetime spectra, indicating unburned oxygen close to the centre of the ejecta. Most explosion models for SNe Ia cannot explain the narrow [O I] emission. Here, we present hydrodynamic explosion and radiative transfer calculations showing that the violent merger of two carbon-oxygen white dwarfs of 0.9 and $0.76 \mathrm{M}_{\odot}$, respectively, adequately reproduces the early-time observables of SN 2010lp. Moreover, our model predicts oxygen close to the centre of the explosion ejecta, a pre-requisite for narrow [O I] emission in nebular spectra as observed in SN 2010lp.

Subject headings: supernovae: individual (SN 2010lp) — hydrodynamics — binaries: close — radiative transfer

\section{INTRODUCTION}

Type Ia supernovae (SNe Ia) form a relatively homogeneous class of objects. It is widely accepted that they result from thermonuclear explosions of carbon-oxygen (CO) white dwarfs (WDs) in binary systems (see e.g. Hillebrandt \& Niemeyer 2000). However, in the absence of direct progenitor detections (Li et al. 2011; Bloom et al. 2012) both the exact nature of the progenitor systems (double- versus single-degenerate) and the details of the explosion mechanism are not well known (Hillebrandt \& Niemeyer 2000). There is now indirect observational evidence (Patat et al. 2007; Li et al. 2011; Bloom et al. 2012, Dilday et al. 2012; González Hernández et al. 2012 Schaefer \& Pagnotta 2012; Shappee et al.|2013) that more than one progenitor type may be responsible for the bulk of normal SNe Ia (e.g. Hillebrandt et al. 2013 , for a review).

A promising method to investigate the nature of SNe Ia is the comparison of theoretical explosion models to observed SN lightcurves and spectra. This has been used extensively in the past (e.g. Höflich \& Khokhlov 1996. Kasen et al. 2009, Sim et al. 2010). However, it can be very difficult to discriminate between different progenitor models for normal SNe Ia from optical lightcurves and spectra alone (Röpke et al. 2012). Nevertheless, specific progenitor and explosion models have been identified for some of the peculiar sub-classes of SNe Ia, like e.g. the subluminous 2002cx-like SNe (Jordan et al. 2012, Kromer

${ }^{1}$ Max-Planck-Institut für Astrophysik, Karl-SchwarzschildStr. 1. 85748 Garching. Germany

${ }^{2}$ The Oskar Klein Centre, Stockholm University, AlbaNova, SE-106 91 Stockholm. Sweden

'Heidelberger Institut für Theoretische Studien, SchlossWolfsbrunnenweg 35, 69118 Heidelberg, Germany

${ }^{4}$ Departamento de Ciencias Fisicas, Universidad Andres Bello, Avda. Republica 252, Santiago, Chile

Institut für Theoretische Physik und Astrophysik, Universität Würzburg, Emil-Fischer-Str. 31, 97074 Würzburg, Germany

${ }^{\circ}$ Astrophysics Research Centre, School of Mathematics and Physics, Queen's University Belfast, Belfast B'T7 INN, UK et al. 2013).

In a companion paper, Pignata et al. (in prep.) present observations of SN 2010lp, a peculiar subluminous SN Ia in NGC 1137 (distance modulus $\mu=33.03 \mathrm{mag}$, redshift $z=0.010, E(B-V)=0.21 \mathrm{mag}$ assumed). At early times this SN has similar brightness and spectra to the subluminous 1991bg-like SNe (Filippenko et al. 1992: Leibundgut et al. 1993) and, like those, does not show double-peaked I-band lightcurves. However, the lightcurve evolution of SN 2010lp is much slower than in 1991 bg-like SNe. Pignata et al. (in prep.) e.g. derive a $\Delta m_{15}(B)$ of 1.24 for SN 2010lp, while typical 1991bg-like SNe have a $\Delta m_{15}(B)$ of $\sim 1.9$ (Taubenberger et al. 2008).

Moreover, narrow [O I] emission has been identified in a nebular spectrum of SN 2010lp (Taubenberger et al. 2013), indicating a concentration of unburned material near the centre of the ejecta. This is very challenging for most current explosion scenarios of SNe Ia discussed in the literature. Neither Chandrasekhar-mass $\left(M_{\mathrm{Ch}}\right)$ delayed detonations (e.g. Seitenzahl et al. 2013) nor sub- $M_{\mathrm{Ch}}$ double detonations and edge-lit detonations (Nomoto 1982, Livne 1990; Fink et al. 2010) are expected to leave $\mathrm{O}$ in the central ejecta, since the densities there are so high that almost all the fuel is completely burned to iron-group elements (IGEs). In contrast, in $M_{\mathrm{Ch}}$-deflagrations the turbulent burning leads to a wellmixed ejecta structure, i.e. $\mathrm{O}$ is present down to the centre of the ejecta (e.g. Ma et al. 2013; Fink et al. 2013). As shown by Kozma et al. (2005), [O I] emission can be present in such models. However, since $\mathrm{O}$ and IGEs are abundant over a wide velocity range, the predicted [O I] feature is broad, unlike the narrow feature observed in SN 2010lp. Taubenberger et al. (2013) pointed out that an ejecta structure similar to that of the violent merger of a 1.1 and a $0.9 \mathrm{M}_{\odot} \mathrm{WD}$ as presented in Pakmor et al. (2012b) might produce narrow [O I] emission in a small region close to the centre of the ejecta. The particular $1.1+0.9 \mathrm{M}_{\odot}$ merger of Pakmor et al. (2012b), however, is significantly too bright to be a good match to SN 2010lp.

Here, we present a hydrodynamic simulation of the vi- 
olent merger of two CO WDs of 0.9 and $0.76 \mathrm{M}_{\odot}$, respectively. By calculating synthetic observables from radiative transfer simulations, we find that this model reproduces the observed early-time properties of SN 2010lp extremely well. Moreover, the explosion ejecta contain $\mathrm{O}$ near the centre, which might potentially lead to [O I] emission in nebular spectra as observed in SN 2010lp.

The Letter is organized as follows. In Section 2 we describe the progenitor system and the hydrodynamic explosion simulation of our model. In Section 3 we present the detailed chemical composition of the explosion ejecta, which we then use in Section 4 to obtain synthetic observables and compare them with SN 2010lp. In Section 5 we discuss our findings before drawing conclusions in Section 6.

\section{MERGER AND EXPLOSION}

Pakmor et al. (2011) used the smoothed-particle hydrodynamics (SPH) code stellar-GADGET (Pakmor et al. 2012a: Springel 2005), to study the inspiral of various pairs of low-mass WDs with different mass ratios. Including a 13-isotope $\alpha$-network, they investigated whether the mergers of these binaries reach sufficiently high temperature and density that the system could detonate. Here, we simulate the explosion of the lowest-mass system for which they found that a detonation is possible, a pair of a 0.9 and a $0.76 \mathrm{M}_{\odot} \mathrm{WD}$.

We take the hydrodynamic structure of the merger at the time of detonation initiation as obtained by Pakmor et al. (2011) and use the LEAFs code (Reinecke et al. 2002) to model the propagation of the detonation through the merged object. LEAFS applies the levelset technique (Reinecke et al. 1999) to model detonation fronts (Fink et al. 2010). To keep track of the rapidly streaming ejecta, we use the expanding-grid technique of Röpke (2005).

The full evolution of our model from the beginning of the SPH inspiral simulation through detonation initiation to homologous expansion is shown in Fig. 1. At the start $(t=0 \mathrm{~s})$, the binary system has an orbital period of $\sim 36$ s on a circular co-rotating orbit. As shown by Pakmor et al. (2012a), this relatively tight orbit has no significant effect on the detonation initiation since, to the accuracy afforded by current simulations, resolution is the limiting factor. In the following $60 \mathrm{~s}$ the primary (more massive) WD accretes matter from the tidally deformed secondary WD. Owing to the high accretion rate, material on the surface of the primary WD is compressed and heated. In the hottest regions, C burning is ignited, and most of the $\mathrm{C}$ is burned in a local thermonuclear runaway that reaches $T=2.67 \times 10^{9} \mathrm{~K}$ and $\rho=1.91 \times 10^{6} \mathrm{~g} \mathrm{~cm}^{-3}$ at $72 \mathrm{~s}$ (black cross in Fig. 1).

We assume that a detonation forms at this instant (Seitenzahl et al. 2009) and map the merger to a uniform $768^{3}$ Cartesian grid to model the detonation with LEAFS. Within the mapping process $0.027 \mathrm{M}_{\odot}$ are lost since some SPH particles lie outside the selected box size of $4 \times 10^{9} \mathrm{~cm}$. However, the initial density of this material is so low that it will not be burned and neither affects the dynamics of the ejecta nor the synthetic observables.

On the grid the detonation is ignited in a spherical bubble of radius $3 \times 10^{7} \mathrm{~cm}$ around the hottest cell. This ignition regions contains 799 cells and consists mainly of $\mathrm{O}$ and intermediate-mass elements due to the preced- ing $\mathrm{C}$ burning. Within $2 \mathrm{~s}$ after detonation initiation $(74 \mathrm{~s})$, almost all material is burned and the explosion ejecta are unbound (energy release from nuclear burning: $1.4 \times 10^{51} \mathrm{erg}$ ). At $172 \mathrm{~s}$ (corresponding to $100 \mathrm{~s}$ after detonation initiation) the ejecta are streaming freely with an asymptotic kinetic energy of $1.1 \times 10^{51} \mathrm{erg}$.

\section{NUCLEOSYNTHESIS}

To obtain the detailed chemical composition of the explosion ejecta, we perform a nucleosynthesis postprocessing calculation with a 384-isotope nuclear network (Travaglio et al. 2004). As input for this calculation we use $10^{6}$ Lagrangian tracer particles for which we recorded the thermodynamic trajectories throughout the hydrodynamic explosion simulation. For the initial composition of the tracer particles we take $50 \% \mathrm{O}$ and $48.29 \% \mathrm{C}$ (by mass). Assuming that the main-sequence progenitor had a solar metallicity, the remaining $1.71 \%$ are distributed according to the solar values of Asplund et al. (2009) for all elements but $\mathrm{H}$ and $\mathrm{He}$. To account for core Heburning all primary $\mathrm{C}, \mathrm{N}, \mathrm{O}$ was converted to ${ }^{22} \mathrm{Ne}$ (by number).

The final ejecta contain $0.21 \mathrm{M}_{\odot}$ of IGEs, of which $0.18 \mathrm{M}_{\odot}$ are ${ }^{56} \mathrm{Ni}$. The most abundant other species are $\mathrm{O}\left(0.50 \mathrm{M}_{\odot}\right), \mathrm{Si}\left(0.37 \mathrm{M}_{\odot}\right), \mathrm{C}\left(0.21 \mathrm{M}_{\odot}\right)$ and $\mathrm{S}\left(0.14 \mathrm{M}_{\odot}\right)$.

To obtain the spatial distribution of chemical species in the ejecta, the final composition of the tracer particles is mapped on a $200^{3}$ Cartesian grid in asymptotic velocity space. For this, we use an SPH-like algorithm that approximately conserves the integrated yields (see Kromer et al. 2010). As can be seen from Fig. 2, the distribution of the ejecta is rather complex, a direct consequence that the merging binary is quite asymmetric at the time of detonation initiation (72 s in Fig. 1). Since detonations propagate faster at higher densities, the primary WD burns first, producing ashes that consist mainly of iron-group and intermediate-mass elements and some O. As these ashes expand, they wrap around the tidal tail, which once was the secondary WD. In this lowdensity material the detonation propagates more slowly and burning is less complete, leaving a significant amount of unburned $\mathrm{O}$. This $\mathrm{O}$ from the secondary WD stays at low velocities and reaches down to the centre of the ejecta.

\section{COMPARISON WITH OBSERVATIONS}

To obtain synthetic observables for our model, we perform Monte Carlo radiative transfer simulations with the ARTIS code (Kromer \& Sim 2009, Sim 2007). As input for this simulation, we take the density distribution from the hydro model and the composition structure from the mapping of the tracer particles, and rescale both to a $50^{3}$ Cartesian grid. For our simulation we use the atomic data set as described by Gall et al. (2012) and propagate $10^{8}$ photon packets for 111 logarithmically spaced time steps between 2 and $120 \mathrm{~d}$ after explosion. To reduce the computational costs, a grey approximation is used in optically thick cells (cf. Kromer \& Sim 2009), and for $t<3 \mathrm{~d}$ local thermodynamic equilibrium is assumed.

In Fig. 3 we present broad-band lightcurves of our model in comparison to the observed photometry of SN 2010lp (Pignata et al. in prep.). As a consequence of the asymmetric ejecta of our model, the lightcurves 


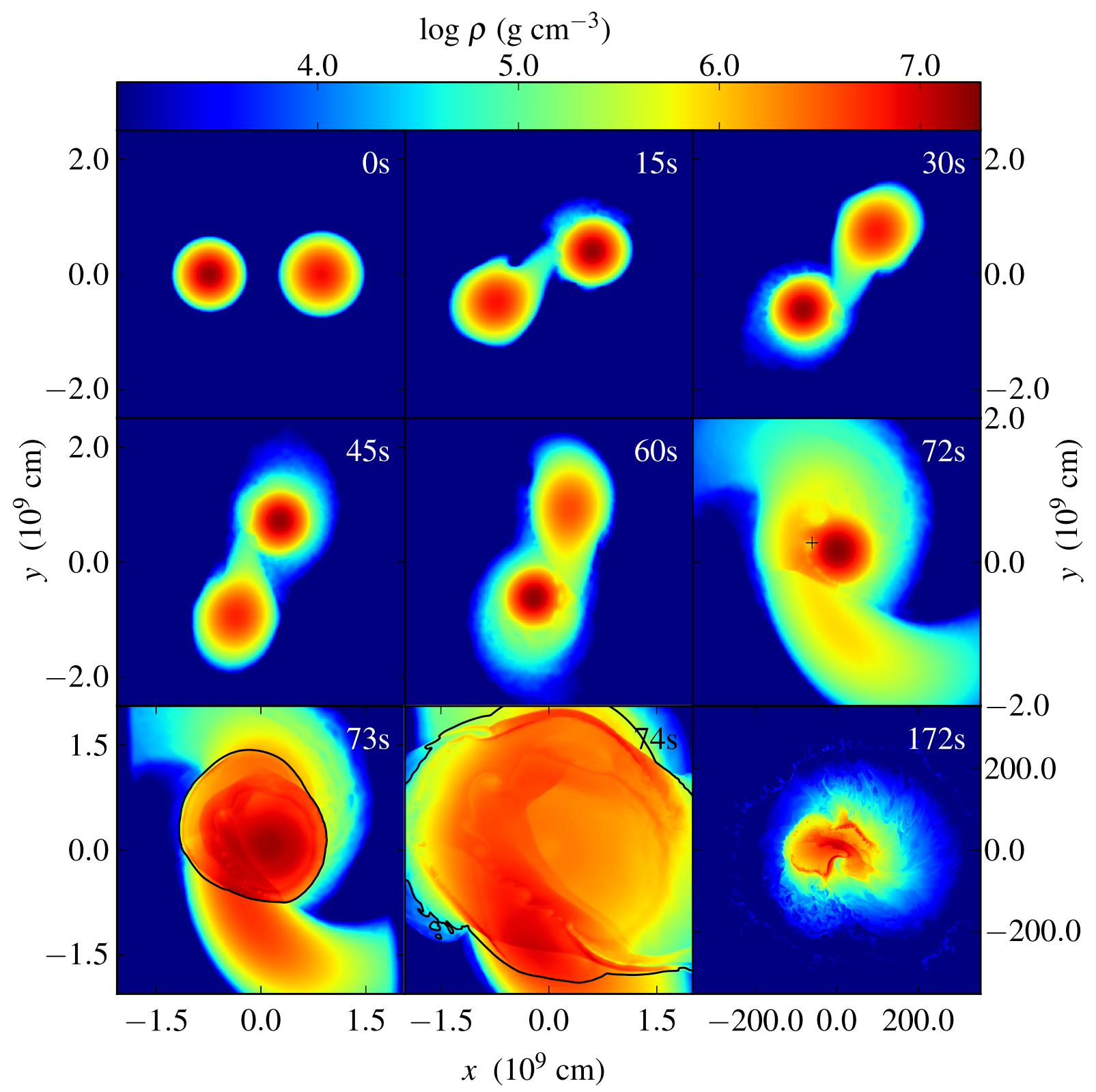

FIG. 1. - Time evolution of our merger model (colour-coded is the logarithm of the density). Initially, the two CO WDs $\left(0.9 \mathrm{M} \odot{ }_{\odot}\right.$ and $0.76 \mathrm{M}_{\odot}$, respectively) orbit each other with a period of $\sim 36 \mathrm{~s}$. The first six panels show the inspiral phase. At $t=72 \mathrm{~s}$, the detonation is ignited (indicated by the crosshairs in the middle right panel). The bottom panels show how the detonation front (black line) propagates. Note that the bottom panels have different colour scales, ranging from $10^{3} \mathrm{~g} \mathrm{~cm}^{-3}$ to $2 \times 10^{6} \mathrm{~g} \mathrm{~cm}^{-3}, 10^{2} \mathrm{~g} \mathrm{~cm}^{-3}$ to $10^{6} \mathrm{~g} \mathrm{~cm}^{-3}$ and $10^{-4} \mathrm{~g} \mathrm{~cm}^{-3}$ to $10 \mathrm{~g} \mathrm{~cm}^{-3}$, respectively.

display a significant spread for different lines-of-sight. In $B$ band, for example, we find peak magnitudes between -18.25 and -17.30 while $\Delta m_{15}(B)$ varies between 0.95 and 1.38. In the redder bands, where the optical depths are smaller, the viewing-angle sensitivity becomes smaller, since a larger fraction of the total ejecta contribute to the emission (compare Kromer \& Sim 2009, Kromer et al. 2010).

Along several of the 100 different lines-of-sight, shown in Fig. 3 (as well as angle-averaged) our synthetic lightcurves show excellent agreement with SN 2010lp, especially in $V$ and $R$. In particular, our model naturally reproduces the low peak brightness, the broad lightcurves and the absence of a secondary maximum in the $I$ band as observed in SN 2010lp.

Fig. 4 shows the spectrum of our model for different lines-of-sight three days before $B$-band maximum in comparison to SN 2010lp at the same epoch (Pignata et al. in prep.). As for the lightcurves, we find a significant sensitivity to viewing-angle effects in the spectrum. For several lines-of-sight as well as the angle-average our synthetic spectrum reproduces SN 2010lp remarkably well. While there are minor discrepancies in the strength of the Ca II (slightly too strong) and Si II (slightly too weak) fea- 


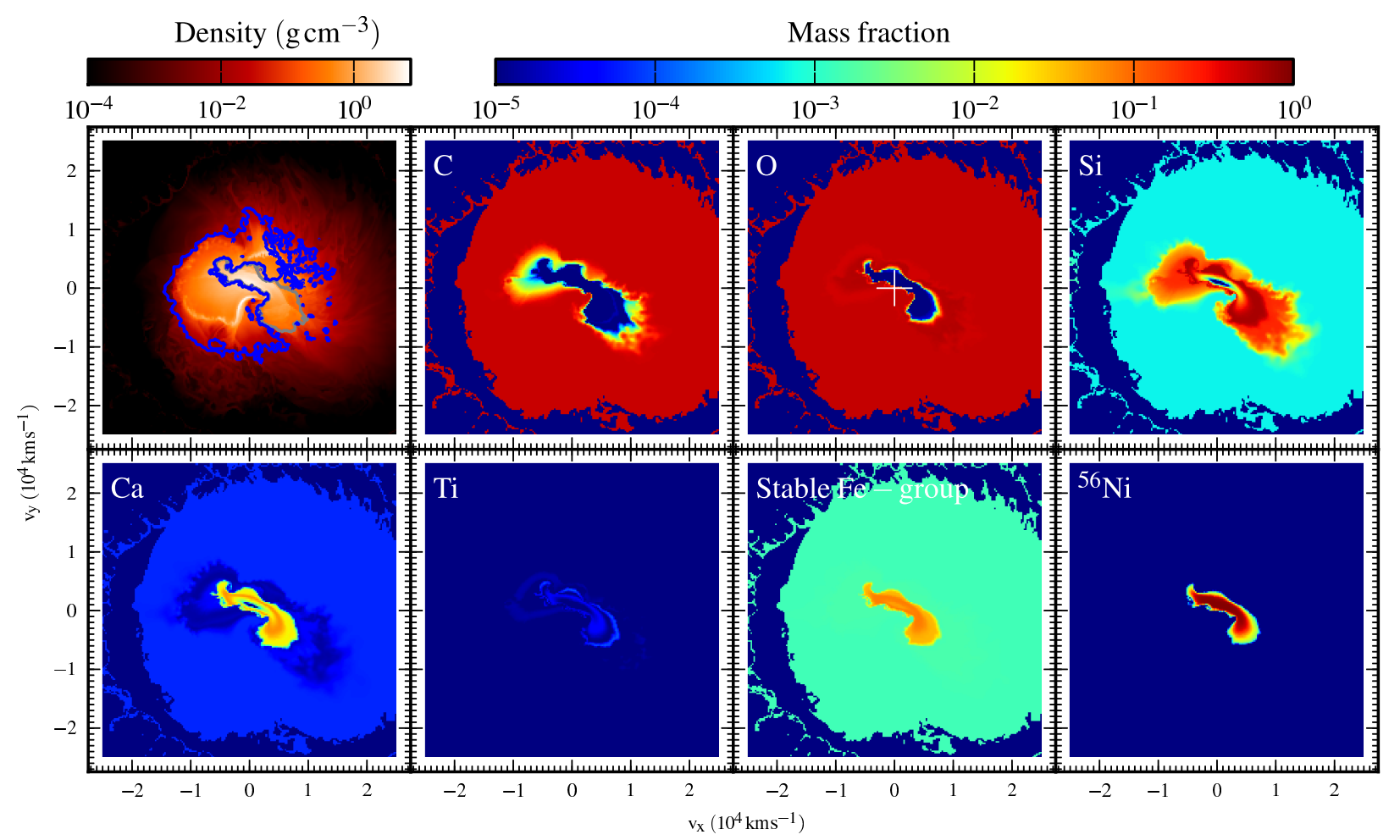

FIG. 2. - Slice through the midplane $(x-y)$ of the simulation volume when the ejecta have reached homologous expansion at $100 \mathrm{~s}$ after the explosion. We show the mass density (top left) and mass fractions of select species as outlined in the different panels. The contours in the density plot indicate regions that contain $>90$ percent material originating from the secondary (blue) or primary (grey) WD.

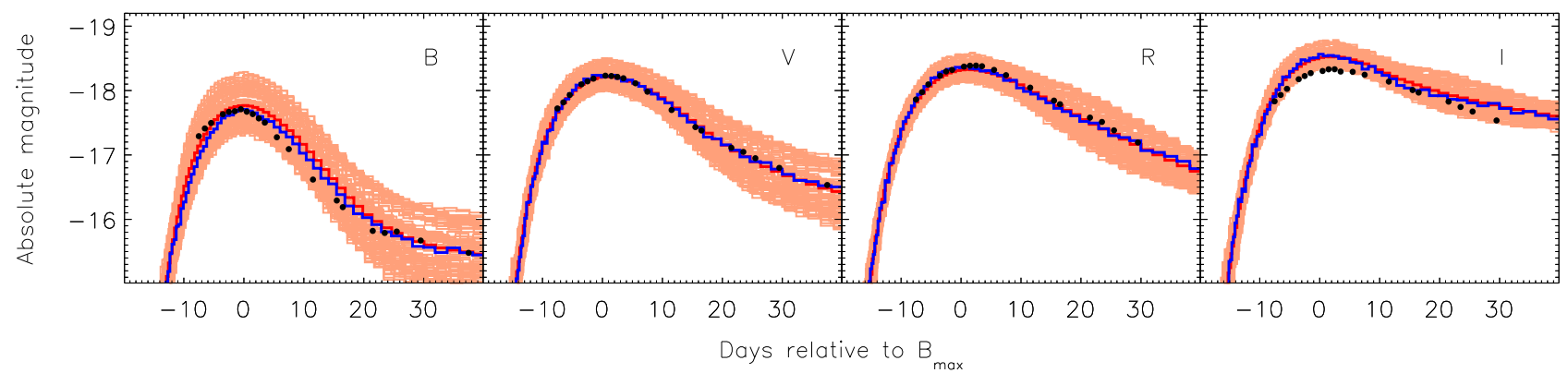

FIG. 3.- Broad-band lightcurves of our model for different filters ( $B, V, R, I$ from left to right, respectively). While 100 light-red lines indicate the spread due to different viewing angles, an individual line-of-sight similar to SN 2010lp is highlighted in blue (the dark-red line shows the angle-average). Time is given relative to $B$-band maximum. For comparison, the observed photometry of SN 2010lp (Pignata et al. in prep.) is overplotted as black circles.

tures, our model clearly shows the hallmark features of 1991bg-like SNe which are also found in SN 2010lp: the broad Ti II absorption trough between 4000 and $4400 \AA$ the relatively strong O I triplet $\lambda \lambda 7772,7774,7775$ and low velocities in the observed absorption features compared to normal SNe Ia. In fact, the velocities of our spectra are even slightly lower than those observed in SN 2010lp.

Regarding the observed [O I] emission in a late-time spectrum of SN 2010lp (Taubenberger et al. 2013), we cannot make a direct prediction for our model at the moment. Our multi-dimensional radiative transfer code ARTIS does not yet take into account the non-thermal excitation and ionization processes necessary to model nebular spectra. One-dimensional codes do not provide a viable alternative either, since mapping the highly asymmetric ejecta of our merger model to $1 \mathrm{D}$ leads to artificial mixing of chemical species, preventing any useful predic- tion. However, from Fig. 2 it is obvious that our model has $\mathrm{O}$ at low velocities, a pre-requisite for narrow [O I] emission in nebular spectra as observed in SN 2010lp.

\section{DISCUSSION}

In comparison to the $0.9+0.9 \mathrm{M}_{\odot}$ merger of Pakmor et al. (2010), our $0.9+0.76 \mathrm{M}_{\odot}$ model is significantly brighter (Fig. 5). This is due to an increased yield of ${ }^{56} \mathrm{Ni}$ in our model. As discussed by Pakmor et al. (2011), the ${ }^{56} \mathrm{Ni}$ production depends only on the density profile of the primary WD at the time of explosion (for a given mass ratio $q=M_{\text {secondary }} / M_{\text {primary }}$ this translates to a dependence on $M_{\text {primary }}$ ). In our model, with $q=0.84$, the density profile of the primary WD at ignition is almost identical to that of an isolated, hydrostatic WD. In contrast, in the Pakmor et al. (2010) merger, with $q=1$, tidal interactions between primary and sec- 

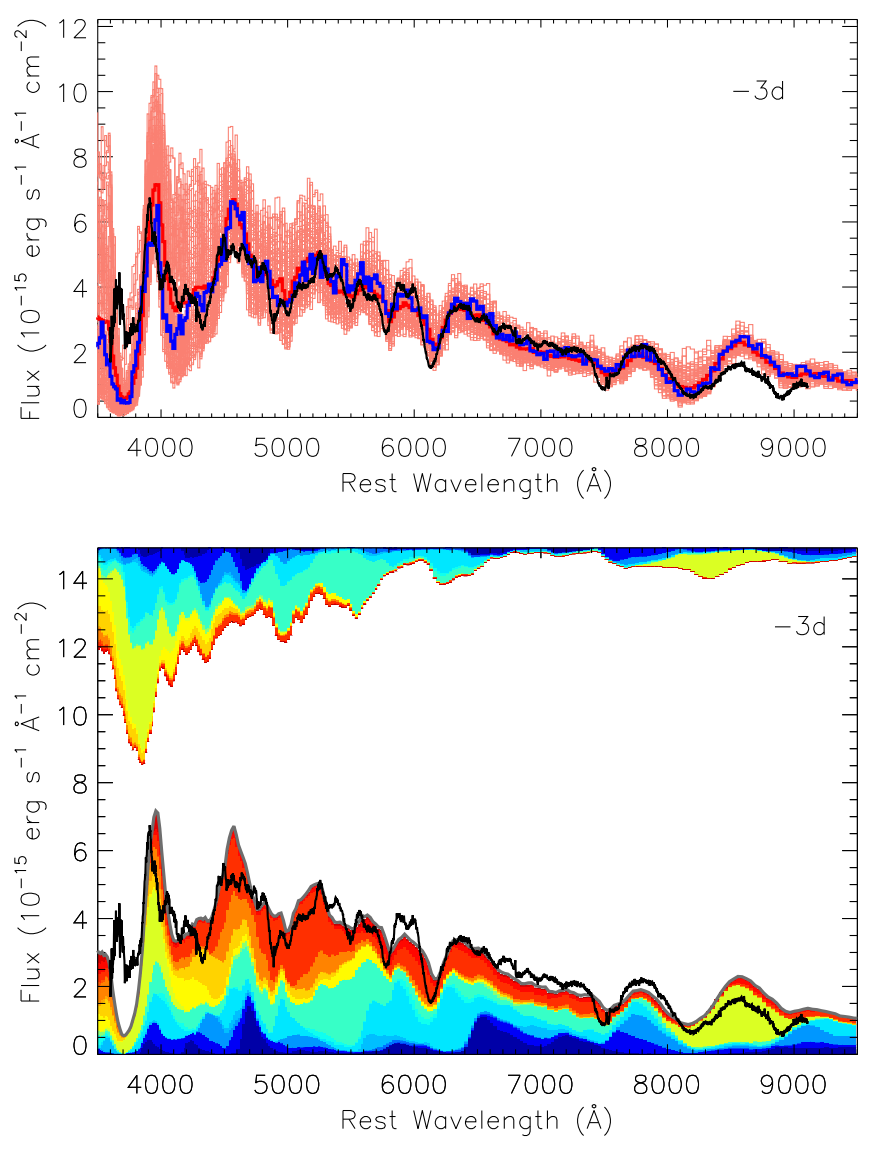

Atomic number

267899101112131415161718192021222324252627282930

FIG. 4. - Top: SN 2010lp about 3 days before maximum (black) compared to synthetic spectra of our merger model as seen along 100 different viewing angles (light-red lines). For comparison, an angle-averaged spectrum (dark red) and an individual line-of-sight (blue) similar to SN 2010lp are shown. Bottom: angle-averaged synthetic spectrum of our merger model (grey) and SN 2010lp (black) about 3 days before $B$-band maximum. For a description of the colour coding see (Kromer et al. 2013), fig. 6.

ondary are strong enough to disturb the density profiles of both WDs, thus reducing their central density and consequently the ${ }^{56} \mathrm{Ni}$ production.

Owing to the low luminosity and the peculiar spectral features of the $0.9+0.9 \mathrm{M}_{\odot}$ merger, Pakmor et al. (2010) proposed their model as a potential progenitor system for $1991 \mathrm{bg}$-like SNe. However, they failed to reproduce the rapid lightcurve evolution typical of those SNe (Fig. 5). Since the opacity in SN ejecta is correlated with their mass, this indicates that the ejecta mass of the Pakmor et al. (2010) model is too large for 1991bg-like SNe.

Maguire et al. (2011) argued that a violent merger of two low-mass CO WDs, as presented by Pakmor et al. (2010), might be the progenitor of PTF10opsanother slowly-declining subluminous SN Ia. The excellent agreement of our model and SN 2010lp strengthens this connection between low-mass CO-CO WD mergers (primary masses $\sim 0.9 \mathrm{M}_{\odot}$ ) and the emerging class of slowly-evolving subluminous SNe Ia like PTF10ops and SN 2010lp.

While binary population synthesis calculations of Ruiter et al. (2013) do not predict a sufficiently large

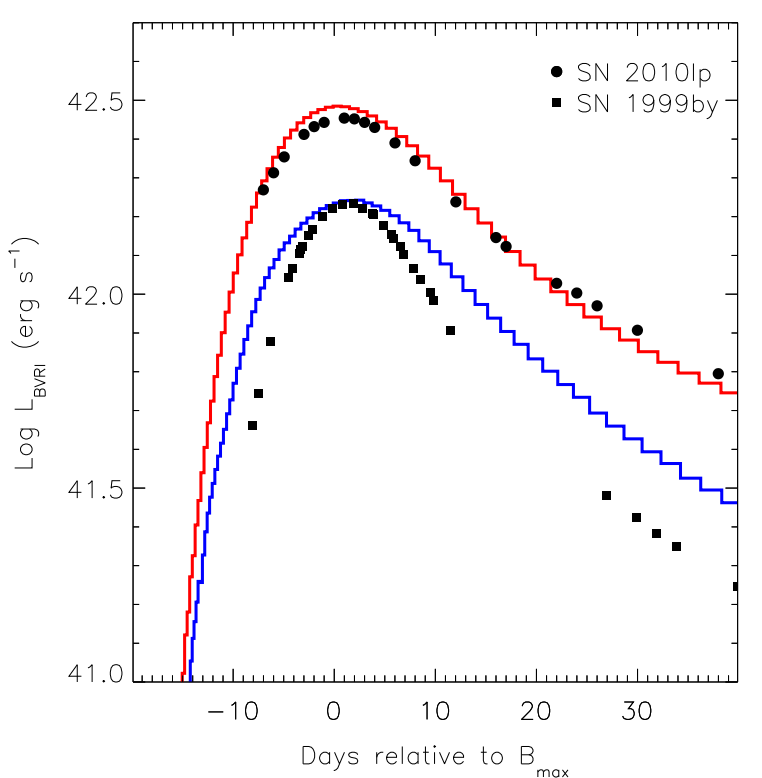

FIG. 5. - BVRI bolometric lightcurves of our merger model (red) and the $0.9+0.9 \mathrm{M}_{\odot}$ merger (blue) of Pakmor et al. (2010). For comparison, pseudobolometric lightcurves constructed from $B V R I$ photometry of SNe 1999by (Garnavich et al. 2004) and 2010lp (Pignata et al. in prep.) are overplotted.

number of low-mass CO-CO mergers to account for the observed number of all faint SNe Ia (see their Fig. 5), the number might be sufficient to account for slowlydeclining subluminous $\mathrm{SNe}$ (from the present observational sample their number is significantly smaller than that of $1991 \mathrm{bg}$-like SNe).

1991 bg-like systems, in contrast, require less massive ejecta. Within the helium-ignited violent merger scenario (Pakmor et al. 2013) CO-He WD systems with a CO WD mass of $\sim 0.8-0.9 \mathrm{M}_{\odot}$ might provide appropriate progenitors for this class of objects. According to population synthesis calculations of Ruiter et al. (2011), mass-transferring $\mathrm{CO}-\mathrm{He}$ systems should be abundant in that mass range. However, more detailed studies, in particular explosion simulations and radiative transfer calculations, will be required to investigate the viability of this model.

In agreement with the absence of narrow [O I] lines in nebular spectra of SN 1991bg and SN 1999by, CO-He mergers would not be expected to show [O I] emission at late times, since the central $\mathrm{O}$ in our model originates from the secondary WD.

For $\mathrm{CO}-\mathrm{CO}$ mergers the question of whether lowvelocity [O I] emission may be expected in late-time spectra is more difficult. A first necessary condition for narrow $[\mathrm{OI}$ ] emission is of course the presence of $\mathrm{O}$ close to the centre of the ejecta. This depends on the mass ratio of the binary systems: mergers with $q \approx 1$ do not produce $\mathrm{O}$ in the centre. In such systems the secondary is already almost destroyed at the time the primary explodes. Consequently, the ejecta of the primary are able to push the material of the secondary away. In contrast, in mergers with $q<1$ the secondary is still very compact when the primary explodes, and thus only marginally affected by the ejecta of the primary. Consequently, the O-rich ashes of the secondary WD stay in the central part of the ejecta. 
However, the mere presence of $\mathrm{O}$ close to the centre of the ejecta is not sufficient for low-velocity [O I] emission in nebular spectra. This depends on the exact excitation and ionisation state of the plasma (see Taubenberger et al. 2013 for a detailed discussion). Low-mass violent $\mathrm{CO}-\mathrm{CO}$ mergers with central $\mathrm{O}$, like our model, could show [O I] emission due to their low luminosity (note, however, that the spatial proximity between ${ }^{56} \mathrm{Ni}$ and $\mathrm{O}$ in our model could still lead to ionisation of the $\mathrm{O}$ ). In contrast, massive mergers, like the $1.1+0.9 \mathrm{M}_{\odot}$ merger of Pakmor et al. (2012b) that resembles normal SNe Ia but also contains $\mathrm{O}$ in the central ejecta, are more luminous. Consequently, the central $\mathrm{O}$ may be ionised, leading to no [O I] emission in agreement with late-time observations of normal SNe Ia.

\section{CONCLUSIONS}

We have presented an explosion model of the violent merger of two CO WDs of 0.9 and $0.76 \mathrm{M}_{\odot}$, respectively. In the explosion ejecta we find $\mathrm{O}$ close to the centre - a pre-requisite for narrow [O I] emission in nebular spectra as observed in the subluminous SN Ia 2010lp. Moreover, from radiative transfer simulations we have shown that our model adequately reproduces the observed early-time observables of SN 2010lp - in particular its low luminosity and slowly-evolving lightcurves, but also its colours and peculiar spectral features.

Our findings support the suggestion of Maguire et al.
(2011) that violent mergers of two CO WDs with lowmass primaries $\left(\sim 0.9 \mathrm{M}_{\odot}\right)$ are a better match to the emerging class of slowly-evolving subluminous SNe Ia like PTF10ops and SN 2010lp, rather than to the rapidly declining 1991bg-like SNe as suggested by Pakmor et al. (2010).

The excellent agreement between our explosion model and SN 2010lp demonstrates the power of theoretical explosion models in finding possible progenitors of (peculiar) SNe Ia. In particular the merger scenario, comprising a large variety of possible progenitor properties warrants detailed scrutiny.

This work was supported by the Deutsche Forschungsgemeinschaft via TRR-33 "The Dark Universe", the graduate school GRK-1147 "Theoretical Astrophysics and Particle Physics" and the Emmy Noether Program (RO 3676/1-1). F.K.R. is supported by the ARCHES prize of the German Ministry of Education \& Research, R.P. by the European Research Council (ERCStG EXAGAL-308037) and G.P. from Millennium Center for Supernova Science (P10-064-F), with input from Fondo de Innovación para la Competitividad, del Ministerio de Economia, Fomento y Turismo de Chile. The simulations were carried out at the Jülich Supercomputing Centre (project hmu14).

\section{REFERENCES}

Asplund, M., Grevesse, N., Sauval, A. J., \& Scott, P. 2009, ARA\&A, 47, 481

Bloom, J. S., Kasen, D., Shen, K. J., et al. 2012, ApJL, 744, L17

Dilday, B., Howell, D. A., Cenko, S. B., et al. 2012, Science, 337, 942

Filippenko, A. V., Richmond, M. W., Branch, D., et al. 1992, AJ, 104,1543

Fink, M., Kromer, M., Seitenzahl, I. R., et al. 2013, ArXiv e-prints

Fink, M., Röpke, F. K., Hillebrandt, W., et al. 2010, A\&A, 514, A53

Gall, E. E. E., Taubenberger, S., Kromer, M., et al. 2012, MNRAS, 427, 994

Garnavich, P. M., Bonanos, A. Z., Krisciunas, K., et al. 2004, ApJ, 613, 1120

González Hernández, J. I., Ruiz-Lapuente, P., Tabernero, H. M., et al. 2012, Nature, 489, 533

Hillebrandt, W., Kromer, M., Röpke, F. K., \& Ruiter, A. J. 2013, Frontiers of Physics, 8, 116

Hillebrandt, W. \& Niemeyer, J. C. 2000, ARA\&A, 38, 191

Höflich, P. \& Khokhlov, A. 1996, ApJ, 457, 500

Jordan, IV, G. C., Perets, H. B., Fisher, R. T., \& van Rossum, D. R. 2012, ApJL, 761, L23

Kasen, D., Röpke, F. K., \& Woosley, S. E. 2009, Nature, 460, 869

Kozma, C., Fransson, C., Hillebrandt, W., et al. 2005, A\&A, 437, 983

Kromer, M., Fink, M., Stanishev, V., et al. 2013, MNRAS, 429, 2287

Kromer, M. \& Sim, S. A. 2009, MNRAS, 398, 1809

Kromer, M., Sim, S. A., Fink, M., et al. 2010, ApJ, 719, 1067

Leibundgut, B., Kirshner, R. P., Phillips, M. M., et al. 1993, AJ, 105,301

Li, W., Bloom, J. S., Podsiadlowski, P., et al. 2011, Nature, 480, 348

Livne, E. 1990, ApJL, 354, L53

Ma, H., Woosley, S. E., Malone, C. M., Almgren, A., \& Bell, J. 2013, ApJ, 771, 58

Maguire, K., Sullivan, M., Thomas, R. C., et al. 2011, MNRAS, 418,747

Nomoto, K. 1982, ApJ, 257, 780
Pakmor, R., Edelmann, P., Röpke, F. K., \& Hillebrandt, W. 2012a, MNRAS, 424, 2222

Pakmor, R., Hachinger, S., Röpke, F. K., \& Hillebrandt, W. 2011, A\&A, 528, A117+

Pakmor, R., Kromer, M., Röpke, F. K., et al. 2010, Nature, 463, 61

Pakmor, R., Kromer, M., Taubenberger, S., et al. 2012b, ApJL, 747, L10

Pakmor, R., Kromer, M., Taubenberger, S., \& Springel, V. 2013, ApJL, 770, L8

Patat, F., Chandra, P., Chevalier, R., et al. 2007, Science, 317, 924

Reinecke, M., Hillebrandt, W., \& Niemeyer, J. C. 2002, A\&A, 386,936

Reinecke, M., Hillebrandt, W., Niemeyer, J. C., Klein, R., \& Gröbl, A. 1999, A\&A, 347, 724

Röpke, F. K. 2005, A\&A, 432, 969

Röpke, F. K., Kromer, M., Seitenzahl, I. R., et al. 2012, ApJL, 750, L19

Ruiter, A. J., Belczynski, K., Sim, S. A., et al. 2011, MNRAS, 417,408

Ruiter, A. J., Sim, S. A., Pakmor, R., et al. 2013, MNRAS, 429, 1425

Schaefer, B. E. \& Pagnotta, A. 2012, Nature, 481, 164

Seitenzahl, I. R., Ciaraldi-Schoolmann, F., Röpke, F. K., et al. 2013, MNRAS, 429, 1156

Seitenzahl, I. R., Meakin, C. A., Townsley, D. M., Lamb, D. Q., \& Truran, J. W. 2009, ApJ, 696, 515

Shappee, B. J., Stanek, K. Z., Pogge, R. W., \& Garnavich, P. M. 2013, ApJL, 762, L5

Sim, S. A. 2007, MNRAS, 375, 154

Sim, S. A., Röpke, F. K., Hillebrandt, W., et al. 2010, ApJL, 714, L52

Springel, V. 2005, MNRAS, 364, 1105

Taubenberger, S., Hachinger, S., Pignata, G., et al. 2008, MNRAS, 385, 75

Taubenberger, S., Kromer, M., Pakmor, R., et al. 2013, ApJL, $775, \mathrm{~L} 43$

Travaglio, C., Hillebrandt, W., Reinecke, M., \& Thielemann, F.-K. 2004, A\&A, 425, 1029 